\title{
The Level and Meaning of IL-18 and Other Factors in Peripheral Blood of Patients with OSAHS Under ERAS Management
}

\author{
Zejun Zhou ${ }^{1}$, Fang $\mathrm{Xu}^{2,}$, \\ ${ }^{1}$ Otolaryngology Department, the First Affiliated Hospital of Jinan University, Guangzhou, P.R.China \\ ${ }^{2}$ Medical Examination Department, the First Affiliated Hospital of Jinan University, Guangzhou, P.R.China
}

Email address:

276705030@qq.com (Fang Xu)

*Corresponding author

\section{To cite this article:}

Zejun Zhou, Fang Xu. The Level and Meaning of IL-18 and Other Factors in Peripheral Blood of Patients with OSAHS Under ERAS Management. Science Journal of Public Health. Vol. 6, No. 4, 2018, pp. 116-119. doi: 10.11648/j.sjph.20180604.14

Received: August 13, 2018; Accepted: September 13, 2018; Published: September 18, 2018

\begin{abstract}
Discus the management of Enhanced recovery after surgery, levels and clinical meaning of interleukin IL-18 and IL-6 in peripheral blood of patients with sleep apnea hypopnea syndrome (OSAHS). By ELISA to detect the levels of IL-18 and IL-6 in serum and peripheral blood mononuclear cells (PBMCs) supernatants of OSAHS patients and healthy controls and check the changes in serum IL-18 and IL-6 levels in OSAHS patients before and after Han uvulopalatopharyngoplasty (H-UPPP) treatment. The levels of IL-18 and IL-6 in serum and PBMCs supernatant of OSAHS patients were higher than those of the matched group $(\mathrm{P}<0.05)$. The levels of serum IL-18 and IL-6 increased with the severity of OSAHS, and the levels of IL-18 and IL-6 in serum and PBMCs supernatants were positively correlated $(\mathrm{P}<0.05)$. After 2 months of H-UPPP, the serum levels of IL-18, IL-6 and the index of respiratory disturbance were significantly lower than before the treatment $(\mathrm{P}<0$. 05). The sleep quality and nocturnal hypoxia were significantly improved, and the lowest oxygen saturation was significantly higher than before the treatment $(\mathrm{P}<0.05)$. IL-18 and IL-6 may play an important role in the pathogenesis and prognosis of OSAHS.
\end{abstract}

Keywords: ERAS Management, Sleep Apnea, Hypoventilation Syndrome, IL-6, IL-18

\section{Introduction}

The concept of the Enhanced Recovery After Surgery (ERAS) was first proposed by Professor Henrik Kehlet of the University of Copenhagen in Denmark in 1997, hoping to reduce the physiological and psychological trauma stress of the surgical patients through a series of optimization measures to optimize the perioperative period to achieve rapid recovery [1]. ERAS has been widely used in many fields at present. The obstructive sleep apnea-hypopnea syndrome, OSAHS refers to the repeated collapse of the upper airway during sleep, accompanied by symptoms such as sleep disorder, apnea, hypoxemia, hypercapnia, daytime sleepiness and snoring, which can lead to complications such as cardiovascular disease and seriously harm the health of the people [2]. At present, the pathogenesis of OSAHS is not very clear. Previous studies have shown that inflammatory cytokines such as $\mathrm{C}$ reactive protein are involved, (tumor necrosis factor, TNF- $\alpha$ ), (interleukin, IL)-6 and so on show high expression in the serum of OSAHS patients [3]. In recent years, systematic research on IL-18 and IL-6 has become more and more profound, but there is still little research on its OSAHS disease [4]. The aim of this study is to check the level of IL-18 and IL-6 in the supernatant of peripheral blood mononuclear cells (PBMCs) in serum and peripheral blood mononuclear cells of OSAHS patients and Changes of serum levels of IL-18 and IL-6 before and after treatment with Han-uvulopalatopharyngoplasty (H-UPPP), to explore the role and meaning of IL-18 and IL-6 in OSAHS pathophysiology and clinical practice $[5,6]$.

\section{Materials and Methods}

\subsection{Case Data}

Selecting 64 cases of OSAHS patients diagnosed by 
polysomnography (PSG) were diagnosed in September 2017 2018 June the First Affiliated Hospital of Jinan University otolaryngology head and neck surgery. According to the diagnostic criteria for the diagnosis and treatment of obstructive sleep apnea hypopnea syndrome, the OSAHS patients were divided into 15 cases of mild OSAHS group, 19 cases of moderate OSAHS group and 26 cases of severe OSAHS group, including 33 male, 27 female and $32 \sim 66$ (age $32 \sim 66) .45 .26+6.59$ years old, all of them were treated with H-UPPP. Another 20 healthy volunteers (all normal by PSG test) were selected as control group, including 11 males and 9 females, aged 32 to $64(44.26+7.02)$ years. There was no significant difference in general data among groups.

\subsection{Method}

\subsubsection{Specimens Collected}

Specimens were collected from each group after the completion of polysomnography on the next day after the completion of the morning and space-time abdominal extraction of venous blood two, of which one ( $3 \mathrm{ml}) 4$ degrees centigrade and $3000 \mathrm{r} /$ min centrifugation $10 \mathrm{~min}$, taking the supernatant and cryopreservation at -80 centigrade; the other $(3 \mathrm{ml})$ was used for the isolation and culture of PBMCs.

\subsubsection{PBMCs Separated}

PBMCs was separated and cultured with $6 \mathrm{ml}$ of venous blood extracted under the aseptic environment, injected into the ACD anticoagulant tube, gently shaking, and adding PBS dilution in proportion to the volume ratio of 1 to 1 at room temperature, and absorbing $6 \mathrm{ml}$ lymphocyte separation liquid (and the volume ratio of the blood specimen before dilution 1 to 1) in the centrifuge tube, and the diluted blood was slow. A centrifuge tube containing lymphocytes was added to the centrifuge tube at 18 $\sim 20$ and $2000 \mathrm{r} / \mathrm{min}$ for $20 \mathrm{~min}$ centrifugation. Carefully drain the PBMCs layer into the new centrifuge tube and mix the PBS solution with $2000 \mathrm{r} / \mathrm{min}$ and $10 \mathrm{~min}$ centrifugation for 2 times. The cells were seeded in the culture plate for $50 \mathrm{ng} / \mathrm{ml}$ PMA and $500 \mathrm{ng} /$ mlIonomycin stimulation experiments. The supernatant of the cell culture medium was collected and stored at -20 .

\subsubsection{Serum, PBMCs Supernatant and H-UPPP Treatment}

Serum, PBMCs supernatant and H-UPPP treatment, IL-18 and IL- 6 were measured by quantitative ELISA method, IL-1 8 and IL-6, IL-6 ELISA kit and IL-18 ELISA reagent box were purchased from Biyun Tian Biotechnology Co., Ltd. Patients in group OSAHS were treated with H-UPPP for 2 months after treatment. The levels of IL-18 and IL-6 in serum were re measured and compared with those before treatment.

Statistical analysis was performed by SPSS 20 software. All normal distribution data were expressed by mean + SD. Single factor analysis of variance (One-way ANOVA) and t test were used. Correlation analysis was conducted by Spearman test with nonparametric statistics.

\section{Results}

\subsection{Comparison of Serum IL-18 and IL-6 Levels in OSAHS Group and Control Group}

Compared with the control group, the serum levels of IL-18 and IL- 6 in the serum were compared with those in the control group. The serum levels of IL-18 and IL-6 in the OSAHS group increased, and the level of IL-18 and IL-6 in the serum increased gradually with the severity of the disease. The difference was statistically significant $(\mathrm{P}<0.05)$. See Table 1

Table 1. Comparison of serum IL-18 and IL-6 levels in OSAHS group and control group.

\begin{tabular}{|c|c|c|c|c|c|}
\hline Item & control group $(n=20)$ & Mild group(n=15) & Moderate group $(n=19)$ & Severe $\operatorname{group}(n=26)$ & F-number \\
\hline IL-18 & $17.25 \pm 4.85$ & $48.18 \pm 7.26^{*}$ & $77.49 \pm 8.33 \#$ & $104.75 \pm 11.47 \Delta$ & $306.51 \pm 36.97$ \\
\hline IL-6 & $4.57 \pm 1.25$ & $19.24 \pm 2.54 *$ & $21.36 \pm 2.66 \#$ & $28.26 \pm 3.07 \Delta$ & $138.26 \pm 14.02$ \\
\hline
\end{tabular}

Comparison with the control group: $* \mathrm{P}<0.05$; Comparison with the Mild group:\#P $<0.05$; Comparison with the Moderate group: $\triangle \mathrm{P}<0.05$

\subsection{Comparison of IL-18 and IL-6 Levels in PBMCs Supernatant of OSAHS Group and Control Group}

The levels of IL-18 and IL-6 in PBMCs supernatant of OSAHS group and control group were compared to detect the level of IL-18 and IL-6 in the supernatant of the cell culture medium after stimulation. The results showed that IL-18 and IL-6 water in PBMCs supernatant of OSAHS group and control group increased significantly, and the difference was statistically significant $(\mathrm{P}<0.01)$. See Table 2 .

Table 2. Comparison of IL-18 and IL-6 levels in PBMCs supernatant of OSAHS group and control group (pg/ ml, mean $\pm S D)$.

\begin{tabular}{lllll}
\hline Item & Control group(n=20) & OSAHS group(n=60) & T-number & P-number \\
\hline IL-18 & $77.24 \pm 8.01$ & $235.41 \pm 25.89$ & -18.45 & $<0.01$ \\
IL-6 & $21.46 \pm 2.28$ & $88.52 \pm 10.37$ & -13.26 & $<0.01$ \\
\hline
\end{tabular}

\subsection{Correlation Analysis Between IL-18 and IL-6 in Serum and Peripheral Blood Mononuclear Cell Culture Medium Supernatant of Group OSAHS}

The levels of IL-18 and IL-6 in serum and in vitro culture of PBMCs supernatant were related to the higher levels of IL-18 and IL-6 in serum and PBMCs culture supernatant in group OSAHS and PBMCs culture. The correlation analysis was made to further study the relationship between the two factors. There was a positive correlation between IL-18 and IL- 6 content in serum and PBMCs medium of OSAHS group. See Figure 1. 
A



B

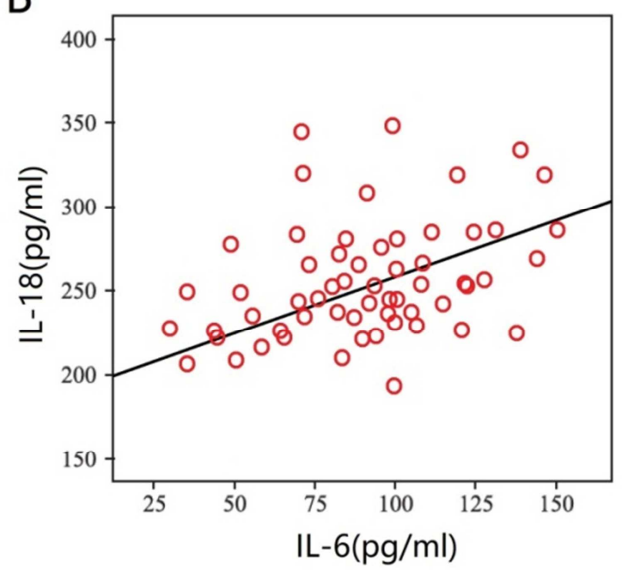

A: OSAHS Group sera;B:OSAHS PBMCs supernatant of the patient

Figure 1. Correlation analysis between IL-18 and IL-6 in serum and peripheral blood mononuclear cell culture medium supernatant of group OSAHS.

\subsection{Changes of Serum IL-18 and IL-6 Levels and Related Indexes in OSAHS Group Before and After Treatment}

After 2 months of H-UPPP operation, the serum IL-18, IL-6 level and respiratory disturbance index (apnea-hypopnea index, AHI) were significantly lower than those before treatment after 2 months of H-UPPP operation. The difference was statistically significant $(\mathrm{P}<0.05)$. The quality of sleep in the patients was significantly improved, and the lowest blood oxygen was found. The degree of saturation (lowest oxyhemoglobin saturation, LSO) was significantly higher than that before treatment $(\mathrm{P}<0.05)$, and the difference was statistically significant $(\mathrm{P}<0.05)$, and the state of hypoxia at night was obviously improved. See Table 3

Table 3. Changes of serum IL-18 and IL-6 levels and related indexes in OSAHS group before and after treatment ( $n=60$, mean $\pm S D)$.

\begin{tabular}{lllll}
\hline Item & Control group(n=20) & OSAHS group $(\mathbf{n}=\mathbf{6 0})$ & T-number & P-number \\
\hline IL-18(pg/ml) & $95.36 \pm 8.26$ & $38.41 \pm 4.26$ & 16.28 & $<0.05$ \\
IL-6(pg/ml) & $26.35 \pm 2.39$ & $10.29 \pm 1.32$. & 14.77 & $<0.05$ \\
AHI(times/h) & $36.55 \pm 3.87$ & $10.54 \pm 1.26$ & 19.57 & $<0.05$ \\
LSO(\%) & $77.41 \pm 9.21$ & $82.34 \pm 10.10$ & -5.26 & $<0.05$ \\
\hline
\end{tabular}

\section{Discussion}

The communication and cooperation between surgeons, anesthesiologists and nurses is the key to the success of ERAS [7]. The perioperative management of ERAS is very different from traditional methods [8]. It is very necessary to inform patients and their families about perioperative treatment [9]. It can reduce the anxiety and fear of patients, so as to better cooperate with the implementation of ERAS [10]. ERAS emphasizes that intestinal preparation is simplified so as to maintain the original intestinal microenvironment and facilitate rapid recovery of intestinal function after operation [11]. Studies have shown that simplified bowel preparation does not increase postoperative anastomotic leakage and postoperative infection rate [12]. ERAS emphasizes effective postoperative analgesia, usually using epidural analgesia pump, and does not affect intestinal function recovery [13]. In respiratory sleep disorders, OSAHS is the most common, which can cause the human body to be at different levels of hypoxic condition. Chronic intermittent hypoxia and hypercapnia can cause increased sympathetic excitability, oxidative stress and inflammation [14]. Previous studies have shown that OSAHS is a chronic inflammatory process mediated by a variety of inflammatory cytokines [15]. The results of this study showed that the content of IL-18 and IL-6 in the serum of OSAHS patients was significantly higher than that of the control group, suggesting that IL-18 and IL-6 may participate in the occurrence of OSAHS together. At the same time, the level of both of them increased with the increase of the severity of OSAHS, suggesting that IL-18 and IL-6 play an important role in the dynamic course of OSAHS.

IL-18, as an important proinflammatory factor, has a strong role in recruiting and activating neutrophils, which stimulates the activation of $\mathrm{T}$ cells and promotes the maturation of dendritic cells [16]. It also mediates the release of a variety of inflammatory mediators and induces inflammatory reactions in the body. It can increase the expression of inflammatory cytokines such as IL-18 and IL-6 and produce synergistic amplification [15]. IL-6 is a member of the IL-12 family, secreted mainly by activated macrophages and dendritic cells, and has an important role in the maintenance of Th17 cell activation [18]. It stimulates the proliferation of Th17 cells and the secretion of IL-18, and promotes the production of chronic inflammatory factors and proinflammatory chemokines in activated Th17 cells. This study showed that the levels of IL-18 and IL-6 in serum and cultured PBMCs supernatants of OSAHS patients were higher than those in the control group, and the serum level of IL-18 and IL-6 in the 
supernatant of PBMCs culture liquid was positively correlated, which showed that there was a certain correlation between IL-18 and IL-6 in the course of OSAHS, and the relationship between them was close [19]. In this study, the serum IL-18 and IL-6 levels of patients in group OSAHS after 2 months of operation were significantly lower than those before the treatment. It was similar to other commonly used evaluation indicators such as AHI and LSO monitoring results, indicating that the level of IL-18 and IL-6 can reflect the clinical process and prognosis of OSAHS disease to a certain extent [20]

\section{Conclusion}

This study suggests that under the management of EARS, IL-18 and IL-6 may participate in the occurrence and development of OSAHS and affect its inflammatory response. At the same time, the dynamic level changes of IL-18 and IL-6 can also be used as a new index for clinical observation of the progression of OSAHS, and further improve the clinical diagnosis of OSAHS.

\section{References}

[1] Yilmaz G, Akca A, Aydin N. Enhanced recovery after surgery (ERAS) versus conventional postoperative care in patients undergoing abdominal hysterectomies. Ginekologia polska. 2018; 89(7):351-6.

[2] Tang XM, Tang LYQ, Li JS. [Sparkplug the construction of enhanced recovery after surgery--promote reform of clinic education]. Zhonghua yi xue za zhi. 2018; 98(28):2217-9.

[3] Zhang T, Zhang C, Chen RX, Shao B, Liu GA. Correlation between coronary artery lesion quantitative score and OSAHS and relative risk factors. European review for medical and pharmacological sciences. 2018; 22(5):1415-20.

[4] Zhang H, Yang F, Guo Y, Wang L, Fang F, Wu H, et al. The contribution of chronic intermittent hypoxia to OSAHS: From the perspective of serum extracellular microvesicle proteins. Metabolism: clinical and experimental. 2018; 85:97-108.

[5] Mei HF, Poonit N, Zhang YC, Ye CY, Cai HL, Yu CY, et al Activating adenosine A1 receptor accelerates PC12 cell injury via ADORA1/PKC/KATP pathway after intermittent hypoxia exposure. Molecular and cellular biochemistry. 2018; 446(1-2):161-70.

[6] Lu HY, Wang W, Zhou Z, Liu CY, Liu Y, Xiao W, et al. Treatment of obstructive sleep apnoea-hypopnea syndrome by mandible advanced device reduced neuron apoptosis in frontal cortex of rabbits. European journal of orthodontics. 2018; 40(3):273-80

[7] Crosson JA. Enhanced Recovery After Surgery-The Importance of the Perianesthesia Nurse on Program Success. Journal of perianesthesia nursing: official journal of the American Society of PeriAnesthesia Nurses. 2018; 33(4):366-74.

[8] Joliat GR, Sauvain MO, Petermann D, Halkic N, Demartines N, Schafer M. Surgical site infections after pancreatic surgery in the era of enhanced recovery protocols. Medicine. 2018; 97(31):e11728.
[9] Estebe JP. Intravenous lidocaine. Best practice \& research Clinical anaesthesiology. 2017; 31(4):513-21.

[10] Dumestre DO, Redwood J, Webb CE, Temple-Oberle C. Enhanced Recovery After Surgery (ERAS) Protocol Enables Safe Same-Day Discharge After Alloplastic Breast Reconstruction. Plast Surg (Oakv). 2017; 25(4):249-54.

[11] Taniguchi H, Sasaki T, Fujita H, Kobayashi H, Kawasaki R, Ogata $\mathrm{T}$, et al. Effects of goal-directed fluid therapy on enhanced postoperative recovery: An interventional comparative observational study with a historical control group on oesophagectomy combined with ERAS program. Clinical nutrition ESPEN. 2018; 23:184-93.

[12] Sayed E, Gaballah K, Younis E, Yassen K, El-Einen AK. The effect of intravenous infusion of $\mathrm{N}$-acetyl cysteine in cirrhotic patients undergoing liver resection: A randomized controlled trial. Journal of anaesthesiology, clinical pharmacology. 2017; 33(4):450-6.

[13] Li H, Chen Z. Application of rehabilitation medicine in enhanced recovery after surgery. Journal of Zhejiang University Medical sciences. 2017; 46(6):675-8.

[14] Meccariello G, Cammaroto G, Montevecchi F, Hoff PT, Spector ME, Negm H, et al. Transoral robotic surgery for the management of obstructive sleep apnea: a systematic review and meta-analysis. Eur Arch Otorhinolaryngol. 2017; 274(2):647-53.

[15] Jia L, Fan J, Cui W, Liu S, Li N, Lau WB, et al. Endothelial Cell-Derived Microparticles from Patients with Obstructive Sleep Apnea Hypoxia Syndrome and Coronary Artery Disease Increase Aortic Endothelial Cell Dysfunction. Cellular physiology and biochemistry: international journal of experimental cellular physiology, biochemistry, and pharmacology. 2017; 43(6):2562-70.

[16] Folk D, D'Agostino M. Transoral robotic surgery vs. endoscopic partial midline glossectomy for obstructive sleep apnea. World journal of otorhinolaryngology - head and neck surgery. $2017 ; 3(2): 101-5$.

[17] Al-Abed M, Antich P, Watenpaugh DE, Behbehani K. Phantom study evaluating detection of simulated upper airway occlusion using piezoelectric ultrasound transducers. Computers in biology and medicine. 2017; 89:325-36.

[18] Basunia M, Fahmy SA, Schmidt F, Agu C, Bhattarai B, Oke V, et al. Relationship of symptoms with sleep-stage abnormalities in obstructive sleep apnea-hypopnea syndrome. Journal of community hospital internal medicine perspectives. 2016;6(4):32170.

[19] Patel AV, Hwang D, Masdeu MJ, Chen GM, Rapoport DM, Ayappa I. Predictors of response to a nasal expiratory resistor device and its potential mechanisms of action for treatment of obstructive sleep apnea. Journal of clinical sleep medicine: JCSM: official publication of the American Academy of Sleep Medicine. 2011; 7(1):13-22.

[20] Callahan CY, Norman RG, Taxin Z, Mooney AM, Rapoport DM, Ayappa I. Multinight recording and analysis of continuous positive airway pressure airflow in the home for titration and management of sleep disordered breathing. Sleep. 2013; 36(4):535-45F. 